\title{
Radon, Baire, and Borel measures on compact spaces. II
}

\author{
H. H. SCHAEFER \\ (Received October 18, 1988)
}

\section{Introduction}

This paper is a direct sequel to [8]. Its prime purpose is to discuss, as transparently as possible and from the functional analytic angle, the various types of measures - their mutual relation and basic properties - that arise in the theory of integration on compact spaces. This is done through a novel approach which the author believes will help to better understand the cross-relations between the set theoretic and functional analytic aspects of this area. The approach is based on imbedding the space $B(X)$ of (real, bounded) Borel functions on $X$ into the second dual $\bar{C}(X)$ of the space $C(X)$ of real continuous functions, without the a priori use of the Riesz representation theorem. This was done in $[8$, B. 2. 6] ; in the interest of independent readability of this second part, the notation and main result of [8] are recalled below (C. 1). It is certainly possible to extend the present method to locally compact spaces $X$, but assuming $X$ to be compact (and restricting attention to bounded Baire and Borel functions) facilitates the discussion without simplifying it beyond the acceptable.

The main result of Section $C$ below is a topological characterization of regular Borel measures on $X$ (C. 2.3), which yields the Riesz representation theorem as well as several other distinguishing properties of regular Borel measures as fairly immediate consequences; for example, the fact that these measures form a band in the (Banach and order) dual $B(X)^{\prime}$ of $B(X)$ (C. 2.5). Section $\mathrm{D}$ is concerned with various aspects of regularity ; for example, the question if a given Borel measure $\mu$ on $X$ is regular, is already determined by the behavior of $\mu$ on $B_{0}(X)$ (D.1.1), where $B_{0}(X)$ is the first member in the transfinite chain $\left\{B_{\alpha}: \alpha<\omega_{1}\right\}$ of Borel classes whose union is $B(X)$. In this context, we feel that the use of transfinite ordinals in the construction of the spaces of Baire and Borel functions, is of greater intuitive appeal than the usual measurability condition. Perhaps unfortunately, the tool of ordinal numbers has been all but abandoned in favor of the (admittedly often convenient) maximality principle; 
a notable exception is Semadeni's monograph [11]. The final part of Section $\mathrm{D}$ is concerned with a characterization of those compact spaces $X$ for which the canonical imbedding $B(X) \rightarrow \bar{C}(X)$ is surjective.

\section{Topological characterization of regular Borel measures}

C. 1 Preliminaries. We recall from [8] the basic notions used throughout this paper. $X$ always denotes a compact (Hausdorff)space; by $C(X)$, $M(X), \bar{C}(X)$ we denote, respectively, the Banach lattice of continuous real functions on $X$, and its first and second dual which are Banach lattices as well. On $\bar{C}(X)$, we consider the topology $\mathfrak{I}=o(\bar{C}(X), M(X))$ of uniform convergence on the order intervals of $M(X)$. Under this topology, $\bar{C}(X)$ is a complete Lebesgue space (see [1], [5], and A.1) with dual $M(X)$; of course, $\bar{C}(X)$ is Dedekind complete and a Banach lattice under its standard norm.

If $L$ denotes the set (convex cone) of all bounded, lower semicontinuous real functions $\geqq 0$ on $X$ and if $M$ denotes the set (convex cone) of all bounded real functions $\geqq 0$ on $X$, the evaluation map $\phi$ : $C(X)_{+} \rightarrow \bar{C}(X)_{+}$has an extension to $L$ and $M$ defined as follows (Def. A. 2 . 1):

$$
\begin{array}{ll}
\bar{\phi}(g)=\sup \{\phi(f): 0 \leqq f \leqq g, f \in C(X)\} & (g \in L) \\
\phi^{*}(h)=\inf \{\bar{\phi}(g): 0 \leqq h \leqq g, g \in L\} & (h \in M) .
\end{array}
$$

It should be noted that the suprema (respectively, infima) in the definitions of $\bar{\phi}$ and $\phi^{*}$ are limits for the topology $\mathfrak{I}$; the basic properties of $\phi^{*}$ were given in Theorem A.2.4. Now let $R$ denote a Riesz subspace of $\boldsymbol{R}^{X}$, containing 1 , of bounded real functions on $X$ : if $\phi^{*}$ is additive on $R_{+}$, then its linear extension $\widetilde{\phi}: R \rightarrow \bar{C}(X)$ is an isometric Riesz isomorphism for the supremum norm of $R$ and the standard norm of $\bar{C}(X)$, respectively (A. 3.5). It turns out (B. 2.6) that the Riesz spaces $R$ with this property include the space $B(X)$ of all bounded Borel functions on $X$ and that $\tilde{\phi}: B(X) \rightarrow \bar{C}(X)$ has some important additional properties (B. 2.6, B. 2.7). (The space $B(X)$ was defined in B. 2.4 and is identical to the space of bounded, real, Borel measurable functions in the usual sense, cf. B. 3.) As the basis of the present paper, we recall these properties, as follows :

1.1. THEOREM. The map $\phi^{*}: B(X)_{+} \rightarrow \bar{C}(X)$ has a linear extension $\tilde{\phi}: B(X) \rightarrow \bar{C}(X)$ which is an isometric Riesz isomorphism with these properties: $\tilde{\phi}$ maps every bounded, pointwise convergent sequence in $B(X)$ onto $a \mathfrak{T}$-convergent sequence, with preservation of limits; moreover, the 
range $\tilde{\phi}(B(X))$ is a sequentially $\mathfrak{I}$-complete (hence Dedekind $\sigma$-complete) Riesz subspace of $\bar{C}(X)$ which is separated by the set $\Delta=\left\{\delta_{t}: t \in X\right\} \subset$ $M(X)$ of all point functionals.

1.2. Under the mapping $\tilde{\phi}$ defined above, we can and shall identify $B(X)$ with a Riesz subspace of $\bar{C}(X)$; in particular, this identification places $B(X)$ and $M(X)$ in separated duality so that $M(X)$ becomes the topological dual of $(B(X), \mathfrak{T})$. Thus in the sequel we shall consider $B(X)$ a Riesz subspace of $\bar{C}(X)$ and $M(X)$ a (Riesz) subspace of the Banach and order dual $B(X)^{\prime}$.

1. 3 Note. The imbedding map $\tilde{\phi}: B(X) \rightarrow \bar{C}(X)$ is uniquely determined by the properties stated in 1.1 and by the requirement that $\tilde{\phi}$ agrees on $L$ with the map $\bar{\phi}$ defined above. The existence of $\tilde{\phi}$ could also be obtained from the Riesz-Markov representation theorem [4]; however, one purpose of this paper is to avoid that approach and instead obtain the Riesz-Markov theorem as an immediate consequence of the topological characterization of regular Borel measures given below (C. 2 . 3).

C. 2 The Dual of $B(X)$. It is well known [4], [10] that the dual of the Banach lattice $B(X)$ (B.2.5) is the Riesz space of all order bounded linear forms on $B(X)$ and can be identified with the Riesz space of all bounded, finitely additive real set functions on $\mathfrak{B}(X)$ (the Borel field of $X$, B.3). Thus if $\mu \in B(X)^{\prime}$ then $A \rightarrow \mu\left(\chi_{A}\right)$ defines a bounded, finitely additive set function on $\mathfrak{B}(X)$ with values in $\boldsymbol{R}$, and conversely. We shall henceforth not distinguish between these two versions and, for $\mu \in B(X)^{\prime}$, we shall write $\mu(A)=\mu\left(\chi_{A}\right)=\int_{A} d \mu$. Moreover, it is well known and easy to see that under this identification, the countably additive set functions agree with the order $\sigma$-continuous [10] linear forms on $B(X)$.

There is less agreement in the literature on the use of the term

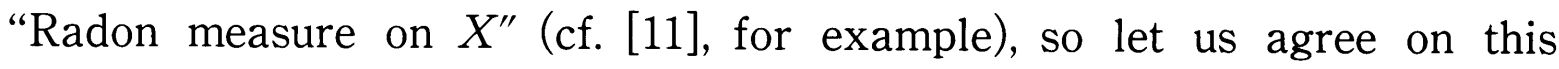
definition.

2.1 Definition. By a Radon measure on $X$, we understand a norm bounded (equivalently, order bounded) linear form on $C(X)$. By a Borel measure on $X$, we understand an order $\sigma$-continuous linear form on $B(X)$.

It is thus clear that $M(X)=C(X)^{\prime}$ and $B(X)^{\prime}$ are Dedekind complete dual Banach lattices; in particular, for $\mu \in B(X)^{\prime}$ it is customary to call $|\mu|$ the total variation of $\mu$. 
2. 2 Definition. Let $\mu \in B(X)^{\prime}$, i. e., let $\mu$ be a bounded, finitely additive set function $\mathfrak{B}(X) \rightarrow \boldsymbol{R}$. $\quad \mu$ is called regular if for each $A \in \mathfrak{B}(X)$,

$$
\sup |\mu|(C)=|\mu|(A)=\inf |\mu|(G)
$$

where $C$ runs through the family of all compact subsets $C$ of $A$ and $G$ runs through the family of all open sets $G$ containing $A$.

We recall that by C.1.2, $B(X)$ is considered a subspace of $\bar{C}(X)$; the topology induced on $B(X)$ by $\mathfrak{I}=o(\bar{C}(X), M(X))$ is again denoted by $\mathfrak{T}$. The following is the announced topological characterization of regular Borel measures on $X$.

2. 3 THEOREM. A linear form $\mu$ on $B(X)$ is a regular Borel measure if and only if $\mu$ is $\mathfrak{I}$-continuous.

PROOF. It is clear that a linear form $\mu$ on $B(X)$ having either of the properties enunciated is bounded, hence in $B(X)^{\prime}$; by Def. 2.2 and because $\mathfrak{I}$ is a locally solid topology [1], [9], it suffices to consider linear forms $\mu \geqq 0$.

Suppose $\mu \geqq 0$ is a regular Borel measure on $X$. Denote by $\mu_{0}$ the restriction of $\mu$ to $C(X)$ (hence, $\mu_{0}$ is a positive Radon measure), and by $\bar{\mu}_{0}$ the unique $\mathfrak{I}$-continuous extension of $\mu_{0}$ to $B(X)$. By C. $1.1, \bar{\mu}_{0}$ is countably additive on $\mathfrak{B}(X)$, and so is $\mu$ by hypothesis. It suffices, therefore, to prove that $\mu$ and $\bar{\mu}_{0}$ agree on the Riesz space $B_{0}(X)$ (in B.2.3, $B_{0}$ was defined as the uniform closure in $\boldsymbol{R}^{X}$ of the Riesz space $L_{0}-L_{0}$ ), for $B(X)$ is the smallest Riesz subspace of $\boldsymbol{R}^{X}$ containing $L_{0}-L_{0}$ and closed under pointwise convergence of uniformly bounded sequences. To this end, it is clearly sufficient to show that $\mu(G)=\bar{\mu}_{0}(G)$ for each open set $G$ $\subset X$. (Recall that $L_{0}$ is the convex conical hull of all $\chi_{G}$, B. 2.2). In turn, for this it suffices to show that $\mu(G)=\sup \left\{\mu(f): 0 \leqq f \leqq \chi_{G}, f \in C(X)\right\}$. Now by regularity, for given $\varepsilon>0$ there exists a compact set $C \subset G$ such that $\mu(G) \leqq \mu(C)+\varepsilon$. By Urysohn's theorem, there exists $f \in C(X), 0 \leqq f$ $\leqq 1$, such that $\chi_{C} \leqq f \leqq \chi_{G}$. It follows that $\mu(G) \leqq \mu(f)+\varepsilon$. Therefore, $\mu$ and $\bar{\mu}_{0}$ agree on all of $B(X)$, and $\bar{\mu}_{0}$ is $\mathfrak{I}$-continuous by definition.

Conversely, suppose that the linear form $\mu \geqq 0$ is $\mathfrak{I}$-contionuous. From C. 1.1 we see that $\mu$ is countably additive, hence a Borel measure; we have to show that $\mu$ is regular. Let $A \subset X$ be any Borel set. By definition of $\phi^{*}$ (see C. 1 above), for a given $\mathfrak{I}$-neighborhood $U$ of 0 there exists $g \in L, \chi_{A} \leqq g$, such that $g-\chi_{A} \in U$. Let $G_{n}:=\left\{t \in X: g(t)>1-n^{-1}\right\}$ : Then $G_{n}$ is open $(n \in N)$ and $\chi_{A} \leqq g \wedge \chi_{G_{n}} \leqq \chi_{G_{n}}$. Since $\chi_{G_{n}}-\chi_{G_{n}} \wedge g \leqq n^{-1} 1$, this latter difference is in $U$ for $n$ large enough. It follows that $\chi_{G_{n}}-\chi_{A} \in$ 
$2 U$ for large enough $n$; since $U$ is arbitrary, we obtain $\chi_{A}=\inf \chi_{G}$ ( $G$ open $\supset A$ ), and this infimum is a limit for the topology $\mathfrak{I}$. Since $\mu$ is $\mathfrak{I}$-continuous, it follows that $\mu$ is regular.

2. 4 Corollary. For a linear form $\mu \in B(X)^{\prime}$ to be a regular Borel measure, it suffices that $\mu$ be $\mathfrak{I}$-continuous on the unit ball of $B(X)$.

In fact, an inspection of the second part of the proof of 2.3 shows that I-continuity of $\mu$ on the set $\{f \in B(X): 0 \leqq f \leqq 1\}$ is sufficient for the conclusion. This means, in particular, that the linear subspace of $\mathfrak{I}$. continuous functionals is norm closed in $B(X)^{\prime}$. Indeed, we can make the following stronger assertion.

2. 5 PRoposition. The Riesz space of all regular Borel measures is a band in $B(X)^{\prime}$. In particular, if $D \neq \emptyset$ is a directed ( $\leqq$ ) norm bounded set of regular Borel measures, then $\mu=\sup D$ is a regular Borel measure.

PROOF. Since $\mathfrak{I}$ is a locally solid topology, it is immediate from 2.3 that the regular Borel measures form an ideal in $B(X)^{\prime}$. On the other hand, since $B(X)$ is an AM-space, the norm of $B(X)^{\prime}$ is additive on $B(X)_{+}^{\prime}[10$, II. 9.1]. Thus $D$ is a Cauchy family for this norm and the assertion follows from 2. 4 .

C. 3. The Riesz-Markov Representation Theorem and Other Consequences of Theorem 2.3. Perhaps the most striking immediate consequence of 2.3 is the following result known as the Riesz (or RieszMarkov) representation theorem (cf. [2], [4], [6], [11]).

3. 1 THEOREM. Every Radon measure $\mu$ on $X$ possesses a unique regular Borel extension $\bar{\mu}$.

Precisely: $\bar{\mu}$ is the unique $\mathfrak{I}$-continuous extension of $\mu \in C(X)^{\prime}$ to $B(X)$, and the map $\mu \rightarrow \bar{\mu}$ is an isometric isomorphism of the Banach lattice $M(X)$ onto the band of regular Borel measures in $B(X)^{\prime}$.

PROOF. The first assertion follows at once from 2.3, since $C(X)$ is a dense Riesz subspace of $B(X)$ for the topology $\mathfrak{I}=o(B(X), M(X))$. Moreover, $\mu \rightarrow \bar{\mu}$ is an isomorphism of Riesz spaces because $\mu \geqq 0$ iff $\bar{\mu} \geqq 0$ (note that $C(X)_{+}$is $\mathfrak{I}$-dense in $B(X)_{+}$), and it is an isometry (for the standard norms of $M(X)$ and $\left.B(X)^{\prime}\right)$ because the unit ball of $C(X)$ is I-dense in the unit ball of $B(X)$ (bipolar theorem, [9]).

3. 2 Note. Since $C(X)$ is a Banach sublattice of $B(X), C(X)^{\prime}=$ $M(X)$ is canonically isomorphic to a quotient of $B(X)^{\prime}$; thus the map $\mu \rightarrow \bar{\mu}$ of 3.1 can be viewed as a lifting of $M(X)$ into $B(X)^{\prime}$. 
3. 3 Proposition. Let $\mu$ be a Borel measure on $X$. If (and only if) $\mu$ is regular, for every non-void directed $(\leqq)$ family $\left\{g_{\alpha}: \alpha \in A\right\}$ in $L$ with least upper bound $g \in L$ we have $\mu(g)=\lim _{\alpha} \mu\left(g_{\alpha}\right)$.

Proof. Let $\Phi_{\alpha}$ denote the set $\left\{f \in C(X): 0 \leqq f \leqq g_{\alpha}\right\}(\alpha \in A)$. Then $\Phi_{\alpha}$ is directed ( $\leqq$ ), and so is $\Phi=\cup_{\alpha} \Phi_{\alpha}$. Now if $f \in C(X), 0 \leqq f \leqq g$, Dini's theorem implies that the directed $(\leqq)$ family $\Phi^{\prime}:=\{f \wedge h: h \in \Phi\}$ converges uniformly to $f$. By definition of the mapping $\bar{\phi}$ (Def. A.2.1 and C. 1), $\Phi \mathfrak{T}$-converges to $g$ and hence $\left\{g_{\alpha}: \alpha \in A\right\} \mathfrak{I}$-converges to $g$. Now if $\mu$ is regular, then by 2.3 we obtain $\mu(g)=\lim _{\alpha} \mu\left(g_{\alpha}\right)$. Conversely, if $\mu$ is a Borel measure, $\mu_{0}$ its restriction to $C(X)$ and $\bar{\mu}_{0}$ the unique regular Borel extension of $\mu_{0}$, then from the equality $\mu(g)=\lim _{\alpha} \mu\left(g_{\alpha}\right)$ above it follows that $\mu$ agrees with $\bar{\mu}_{0}$ on $B_{0}(X)$ and hence, by countable additivity, on all of $B(X)$ (cf. Def. B. 2.4).

3. 4 COROLlary. If $\left\{G_{\alpha}: \alpha \in A\right\}$ is a non-void family of pairwise disjoint open sets and $\mu$ is a regular Borel measure on $X$, then the family $\left\{\mu\left(G_{\alpha}\right): \alpha \in A\right\}$ is summable and, if $G=\cup G_{\alpha}$,

$$
\mu(G)=\sum_{\alpha \in A} \mu\left(G_{\alpha}\right) .
$$

We point out that 3.3 generally fails for uncountable families if their members $g_{\alpha}$ are not supposed to be lower semi-continuous.

3. 5 Proposition. Let $A_{i}(i=1, \cdots, n)$ be Borel sets, let $\mu \geqq 0$ be a regular Borel measure on $X$, and let $\alpha_{i}(i=1, \cdots, n)$ be real numbers such that $\int\left|c_{1} \chi_{A_{1}}+\cdots+c_{n} \chi_{A_{n}}\right| d \mu=0$ implies $c_{1} \alpha_{1}+\cdots c_{n} \alpha_{n}=0$. There exists a bounded Borel function $f$ on $X$ such that

$$
\int_{A_{i}} f d \mu=\alpha_{i} \quad(i=1, \cdots, n) .
$$

ProOF. Denote by $E$ the finite-dimensional vector subspace of $B(X)$ spanned by $\left\{\chi_{A i}: i=1, \cdots, n\right\}$, let $n_{0}=\operatorname{dim} E$, and let $U=\{f \in B(X): \mu(|f|)$ $\leqq 1\}$. The condition $\int\left|c_{1} \chi_{A_{1}}+\cdots+c_{n} \chi_{A_{n}}\right| d \mu=0$ determines a $k$-dimensional $\left(k \leqq n_{0} \leqq n\right)$ subspace $E_{0}$ of $E$ which is the intersection of the null ideal of $U$ with $E$. There exist exactly $n_{0}-k$ independent linear forms on $E$ that vanish on $E_{0}$. By virtue of the hypothesis, a suitable linear combination $\nu$ of these forms satisfies the conditions $\nu\left(\chi_{A_{i}}\right)=\alpha_{i}(i=1, \cdots, n)$, and $\nu$ is bounded on $V=E \cap U$ by some $\beta \in \boldsymbol{R}$. Thus by the Hahn-Banach theorem, there exists a linear extension $\mu_{1}$ of $\nu$ to $B(X)$ such that $\left|\mu_{1}(f)\right| \leqq \beta$ for all $f \in U$; in particular, $\mu_{1}$ is $\mathfrak{I}$-continuous and hence a regular Borel 
measure C. 2.3. Moreover, $\mu_{1} \in \beta U^{\circ}$ where $U^{\circ}$ denotes the polar of $U$ in $M(X)$; but $U^{\circ}=[-\mu, \mu]$ and thus $\mu_{1} \in \beta[-\mu, \mu]$. By the Radon-Nikodym theorem, we have $\mu_{1}=f \mu$ for some $f \in B(X),\|f\| \leqq \beta$. Since $\mu_{1}\left(A_{i}\right)=\alpha_{i}$ $(i=1, \cdots, n)$, the assertion follows.

\section{Regularity of Baire and Borel measures}

D. 1 Regularity of Borel Measures. It was shown in B. 2 that, if $L_{0}$ denotes the convex conical hull of all characteristic functions $\chi_{G}$ of open subsets $G \subset X, L_{0}-L_{0}$ is a Riesz subspace of $\boldsymbol{R}^{X}$ whose uniform closure $B_{0}=B_{0}(X)$ is a Banach lattice containing $C(X)$ (B.2.3). Starting with $B_{0}$, the Borel classes $B_{\alpha}\left(\alpha<\omega_{1}\right)$ of bounded functions were then defined by transfinite induction (B.2.4) in a manner entirely analogous to the customary definition of the Baire classes $\widetilde{B}_{\alpha}$ (B. 1).

1. 1 Proposition. A Borel measure $\mu$ on $X$ is regular if and only if $\mu$ is $\mathfrak{T}$-continuous on $B_{0}(X)$.

Proof. The necessity of the condition is clear from C.2.3. Conversely, if the restriction $\mu_{1}:=\mu \mid B_{0}(X)$ is $\mathfrak{I}$-continuous, then $\mu_{1}$ has a (unique) $\mathfrak{I}$-continuous extension $\bar{\mu}_{1}$ to $B(X)$, which is regular by C.2.3. Since $\mu$ is countably additive (Def. C.2.1), the construction of $B(X)$ implies that $\mu=\bar{\mu}_{1}$, hence that $\mu$ is regular.

1. 2 Note. Since (by definition of $B_{0}$ ) the Riesz space $L_{0}-L_{0}$ is uniformly dense in $B_{0}$, a Borel measure $\mu$ is regular even if its restriction to $L_{0}-L_{0}$ is $\mathfrak{I}$-continuous; in fact, it suffices that $\mu$ be $\mathfrak{I}$-continuous on the unit ball of $L_{0}-L_{0}$ (cf. C. 2.4).

Our next result is a special case of a well known theorem due to Alexandroff [4, III. 5.13]. As before (B. 2) we denote by $\mathfrak{B}(X)$ the $\sigma$-field of all Borel subsets of $X$.

1.3 Proposition. If $\mu$ is a bounded, finitely additive set function on $\mathfrak{B}(X)$ which is regular, then $\mu$ is countably additive (hence a regular Borel measure).

Proof. Let $\mu_{0}$ denote the restriction of $\mu$ to $C(X)$, and let $\bar{\mu}_{0}$ denote the regular Borel extension of $\mu_{0}$ (C. 3.1). Since $\mu$ and $\bar{\mu}_{0}$ are regular set functions, it suffices to show that $\mu$ and $\bar{\mu}_{0}$ agree on all open sets; then $\mu$ and $\bar{\mu}_{0}$ will agree on $\mathfrak{B}(X)$.

Without loss of generality, we can suppose that $\mu \geqq 0$. We show that $\mu(G)=\sup _{A} \mu(f)$, where $A=\left\{f \in C(X): 0 \leqq f \leqq \chi_{G}\right\}$ and $G$ is open. By regularity, we have $\mu(G)=\sup _{B} \mu(C)$ where $B$ is the family of all compact sub- 
sets of $G$. But for each $C \in B$, there exists $f \in A$ such that $\chi_{C} \leqq f \leqq \chi_{G}$ (Urysohn's theorem). Thus $\mu(G)=\sup _{A} \mu(f)$ and hence, $\mu(G)=\bar{\mu}_{0}(G)$.

The following theorem, which is hard to find in the literature, draws on the deeper properties of the space $B(X)$.

1. 4 THEOREM. Let $\left(\mu_{n}\right)$ be a sequence of regular Borel measures such that for each $A \in \mathfrak{B}(X), \lim _{n} \mu_{n}(A)$ exists (in $\left.\boldsymbol{R}\right)$. Then $A \rightarrow \lim _{n} \mu_{n}(A)$ is a regular Borel measure.

PRoOF. It follows from the Nikodym Boundedness Theorem [3, I. 3 Thm. 1] that the sequence $\left(\mu_{n}\right)$ is norm bounded in $B(X)^{\prime}$ (in fact, the space of simple Borel functions is a barreled subspace of $B(X))$; hence, it converges to some $\mu \in B(X)^{\prime}$ for the weak* topology $\sigma\left(B(X)^{\prime}, B(X)\right)$. By a well known theorem of $T$. Ando [10, II. 10.4], $B(X)$ is a Grothendieck space hence, we have $\mu=\lim _{n} \mu_{n}$ for the weak topology of $B(X)^{\prime}$. Since by C. 3.1, the space of regular Borel measures is a norm (and hence weakly) closed subspace of $B(X)^{\prime}$, we conclude that $\mu$ is a regular Borel measure.

D. 2 Baire Measures. The Riesz space $\widetilde{B}(X)$ of all bounded Baire functions on $X$ (B.1) is clearly $\mathfrak{T}$-dense in $B(X)$; as can be seen from B. 1. 3, $\widetilde{B}(X)$ is $\mathfrak{T}$-sequentially closed in $B(X)$. In particular, if two regular Borel measures agree on $\widetilde{B}(X)$ they are identical [6, p. 229].

We recall from B. 3 that the Baire field $\widetilde{B}(X)$ consists of all subsets $A$ $\subset X$ such that $\chi_{A}$ is a Baire function. Just as in the case of $B(X)$ (see C. 2 ), the Banach (and order) dual of $\widetilde{B}(X)$ is the Riesz space $\widetilde{B}(X)^{\prime}$ of all bounded, finitely additive set functions $\widetilde{\mathfrak{B}}(X) \rightarrow \boldsymbol{R}$ (or equivalently, of all order bounded linear forms). The Riesz subspace of all order $\sigma$ continuous linear forms is called the space of (bounded) Baire measures on $X$; i. e., a Baire measure is a countably additive (necessarily bounded) set function $\widetilde{\mathfrak{B}}(X) \rightarrow \boldsymbol{R}$. As before, we identify the set function $\mu$ with the linear form $f \rightarrow \int f d \mu$ it defines on $\widetilde{B}(X)$. It is well known [10, II. 4.3 Cor.] that the Baire measures on $X$ form a band in $\widetilde{B}(X)^{\prime}$.

As agreed upon earlier (C. 1.2) we shall consider $\widetilde{B}(X)$ as a (Dedekind $\sigma$-complete) Riesz subspace of the bidual $\bar{C}(X)$ of $C(X)$; the topology on $\widetilde{B}(X)$ induced by $\mathfrak{I}=o(\bar{C}(X), M(X))$ will again be denoted by $\mathfrak{I}$.

2.1 Proposition. A linear form $\mu \in \widetilde{B}(X)^{\prime}$ is a Baire measure if and only if $\mu$ is $\mathfrak{T}$-continuous.

PROOF. If $\mu$ is $\mathfrak{I}$-continuous then $\mu$ is clearly bounded and, since bounded pointwise convergent sequences in $\widetilde{B}(X)$ converge for $\mathfrak{I}$ (B. 1.3), 
countably additive.

Conversely, let $\mu$ be a Baire measure, $\mu_{0}$ its restriction to $C(X)$, and $\bar{\mu}_{0}$ the unique $\mathfrak{I}$-continuous extension of $\mu_{0}$ to $B(X)$. By 2.3, $\bar{\mu}_{0}$ is a regular Borel measure, hence countably additive. Thus by definition of $\widetilde{B}(X)$ (B. 1$), \mu$ agrees with $\bar{\mu}_{0}$ on $\widetilde{B}(X)$ and is, therefore, I-continuous.

2. 2 Lemma. Let $A \subset X$ be a Baire set, $G$ an open, $F$ a compact subset of $\mathrm{X}$ such that $F \subset A \subset G$. There exists a compact Baire set $\widetilde{F}$ and an open Baire set $\widetilde{G}$ satisfying $F \subset \widetilde{F} \subset A \subset \widetilde{G} \subset G$.

PROOF. As in the proof of B.3.2, it follows by transfinite induction on the Baire order of $A$ (i. e., on the Baire order of $\chi_{A}$, Def. B. 3.1) that there exists a sequence $\left(F_{n}\right)$ of compact subsets of $G$ such that $A \subset \cup_{n} F_{n}$. By Urysohn's theorem, for each $n$ there exists a continuous function $f_{n}$ such that $\chi_{F_{n}} \leqq f_{n} \leqq \chi_{G}$. Now $\widetilde{G}_{n}=\left\{t \in X: f_{n}(t)>0\right\}$ is an open Baire set (because it is an $F_{\sigma}$-set, B. 3.2) for each $n$; hence, $\widetilde{G}:=\cup_{n} \widetilde{G}_{n}$ is an open Baire set satisfying the assertion. The remainder follows by complementation.

2. 3 Definition. A Baire measure $\mu$ on $X$ is called (Baire) regular if for each Baire set $A \subset X$ and each real number $\varepsilon>0$, there exist a compact Baire set $\widetilde{F}$ and an open Baire set $\widetilde{G}$ such that $\widetilde{F} \subset A \subset \widetilde{G}$ and $|\mu|(\widetilde{G} \backslash \widetilde{F})<\varepsilon$.

The following theorem is well known (see, for example, [6, p. 228]) but still somewhat surprising.

2. 4 THEOREM. Every Baire measure on $X$ is (Baire) regular.

PRoof. Let $\mu$ denote a Baire measure on $X$; by Def. 2.3 we can assume that $\mu \geqq 0$. Since $\mu$ is $\mathfrak{T}$-continuous by 2.1 , it has a unique $\mathfrak{I}$. continuous extension $\bar{\mu}$ to $B(X)$; by C.2.3, $\bar{\mu}$ is regular. The assertion follows now immediately from 2.2 and the definitions of regularity and Baire regularity, respectively (C. 2.2, D.2.3).

2. 5 NoTES. Let us remark that for Baire measures, complete analogs of Theorems C. 3. 1 and D. 1.4 are valid; in particular, the band of all Baire measures in $\widetilde{B}(X)^{\prime}$ is Riesz and norm isomorphic to the Banach lattice $M(X)$ of all Radon measures on $X$.

In fact, if $\mu \in M(X)$ then $\mu$ has a unique Baire extension $\tilde{\mu}$ to $\widetilde{B}(X)$, and $\tilde{\mu}$ is the restriction to $\widetilde{B}(X)$ of the unique resular Borel extension $\bar{\mu}$ of $\mu$. In the chain of (isometric) inclusions $C(X) \subset \widetilde{B}(X) \subset B(X)$, each space is $\mathfrak{I}$-dense in its successor, and the same holds for the respective 
unit balls. However, while $\widetilde{B}(X)$ is $\mathfrak{T}$-sequentially closed in $B(X), \widetilde{B}(X)$ is identical with the $\mathfrak{T}$-sequential closure of $C(X)$ in $B(X)$ (or in $\bar{C}(X)$ ).

Thus it is no surprise that a Baire measure-through order $\sigma$ continuity (or equivalently, countable addivity) -is uniquely determined by its restriction to $C(X)$. What is perhaps surprising is the fact that order $\sigma$-continuity of $\mu \in \widetilde{B}(X)^{\prime}$ implies continuity for the topology $\mathfrak{I}$. This, in turn, implies the (Baire) regularity of each Baire measure.

D. 3 Inclusion Relations of $C(X), \widetilde{B}(X), B(X)$, and $\bar{C}(X)$. In the preceding notes, we have considered the chain of inclusions $C(X) \subset \widetilde{B}(X)$ $\subset B(X) \subset \bar{C}(X)$, where $B(X)$ is canonically identified with a Riesz subspace of $\bar{C}(X)$ by virtue of C.1.1, C.1.2. In this final section, we aim at characterizing those compact spaces $X$ for which some or all of these inclusions are not proper.

To characterize the equality $C(X)=\widetilde{B}(X)$, we need this elementary lemma.

3. 1 Lemma. Every infinite, compact space $X$ contains a sequence $\left(F_{n}\right)_{n \in N}$ of pairwise disjoint closed subsets with non-void interior.

PRoOF. If $X$ contains infinitely many isolated points, there is nothing to prove; so let us assume $X$ does not. We construct a sequence $\left(F_{n}\right)$ with the required properties by induction, as follows. Let $t_{1} \in X$; if every compact neighborhood of $t_{1}$ has finite complement, then $t_{1}$ is the unique accumulation point of $X$, contrary to our assumption. Thus there exists a compact neighborhood $F_{1}$ of $t_{1}$ such that $X \backslash F_{1}$ is infinite. Suppose now that $F_{1}, \cdots, F_{n-1}$ are disjoint compact sets, each with non-void interior, such that $G_{n}:=X \backslash \bigcup_{\nu=1}^{n-1} F_{\nu}$ is infinite. Then if $t_{n} \in G_{n}$, there exists a compact neighborhood $F_{n}$ of $t_{n}$ in $G_{n}$ such that $G_{n} \backslash F_{n}$ is infinite; otherwise $t_{n}$ would be the unique accumulation point of the locally compact space $G_{n}$, which is impossible. The sequence $\left(F_{n}\right)$, defined by induction, satisfies the assertion.

3. 2 Proposition. One has $C(X)=\widetilde{B}(X)$ if and only if $X$ is finite.

PROOF. Clearly, we only have to show that the condition is necessary. If $C(X)=\widetilde{B}(X)$ then each bounded Baire function on $X$ is continuous; in particular, each Baire set is open and closed (hence compact). This implies that every family of disjoint non-void open Baire sets is finite; obviously, then, the same holds for any family of disjoint, non-void open subsets of $X$. Thus by $3.1, X$ is finite. 
3. 3 PROPOSITION. The following assertions are pairwise equivalent:

(a) Every Borel subset of $X$ is a Baire set.

(b) Every open subset of $X$ is an $F_{\sigma}$-set.

(c) $\widetilde{B}(X)=B(X)$.

Proof. $\quad(a) \Longrightarrow(c)$ : Since every $f \in B(X)$ is the uniform limit of a sequence of simple Borel (hence Baire) functions, and since $\widetilde{B}(X)$ is closed in $\boldsymbol{R}^{X}$ under the topology of uniform convergence, it follows that $B(X) \subset \widetilde{B}(X)$.

(c) $\Longrightarrow$ (b): If $G$ is open in $X$, it suffices to apply the first part of the proof of 2.2 to $A=G$.

(b) $\Longrightarrow(\mathrm{a})$ : (h) implies that the space $B_{0}(X)$ (Def. B. 2.3) is contained in the first Baire class $\widetilde{B}_{1}(X)$; hence, by B. 2. $4, \mathfrak{B}(X) \subset \widetilde{\mathfrak{B}}(X)$.

3. 4 REMARKS. It is well known [6, p. 218] that separability of $X$ implies $\widetilde{B}(X)=B(X)$; on the other hand, a necessary condition for this equality to hold is that every disjoint family of non-void open subsets of $X$ be countable.

Each of the equivalent assertions of 3.3 implies that every Borel measure on $X$ is regular; it would be interesting to know if this condition actually characterizes the equality $\widetilde{B}(X)=B(X)$.

For a characterization of the equality $B(X)=\bar{C}(X)$, we need another seemingly well known result whose proof is included for completeness.

3. 5 Proposition. The order continuous dual of $B(X)$ is $l^{1}(X)$. Moreover, $B(X)$ is Dedekind complete if and only if $\mathfrak{B}(X)=2^{X}$.

PROOF. Let $\mu$ denote an order continuous linear form on $B(X)$; without loss of generality we assume that $\mu \geqq 0$. If $\chi_{t}$ denotes the characteristic function of the singleton $\{t\} \subset X$, then $\mu\left(\chi_{t}\right)=0$ except for $t \in A$, where $A$ is a countable subset of $X$ (depending on $\mu$ ). If $A_{1}:=X \backslash A$ then $\chi_{A_{1}}=\sup \left\{\chi_{t}: t \in A_{1}\right\}$ and, by order continuity, $\mu\left(A_{1}\right)=0$. Thus $\mu=$ $\sum_{t \in A} a_{t} \delta_{t}$ where $\left(a_{t}\right) \in l^{1}(X)$ with $\|\mu\|=\sum_{t \in X} a_{t}$. Clearly, $l^{1}(X)$ is a Riesz subspace (in fact, a band) in $M(X)$.

Clearly, if $\mathfrak{B}(X)=2^{X}$ then $B(X)$ is Dedekind complete. Conversely, let $B(X)$ be Dedekind complete. If $A$ is any non-void subset of $X$, let $h$ : $=\sup \left\{\chi_{t}: t \in A\right\}$ in $B(X)$. The set $A_{1}:=\{t \in X: h(t)>0\}$ is a Borel set; if $A_{1} \backslash A$ were non-void then $h_{1}=h \wedge \chi_{A_{2}}$, where $A_{2}=A_{1} \backslash\{t\}$ for some $t \in$ $A_{1} \backslash A$, would be an upper bound for all $\chi_{t}(t \in A)$ and strictly less than $h$, which is impossible. Thus $A=A_{1}$ and $\mathfrak{B}(X)=2^{X}$.

Let us recall again that by $\mathfrak{I}$ we denote the weak Riesz topology 
$o(\bar{C}(X), M(X))$ on $\bar{C}(X)$ as well as the induced topology $o(B(X), M(X))$ on $B(X)$ (C. 1.2). A compact space $X$ is scattered [11, 8.5] if $X$ contains no non-void perfect subset, or equivalently, if for some ordinal $\alpha$ the $\alpha$-th derived set $X^{(\alpha)}$ is void.

3. 6 THEOREM. The following assertions are pairwise equivalent :

(a) $B(X)=\bar{C}(X)$

(b) $B(X)$ is Dedekind complete and $X$ is scattered.

(c) Every bounded real function on $X$ is a Borel function and every regular Borel measure on $X$ is purely atomic.

(d) $(B(X), \mathfrak{I})$ is complete.

Moreover, if the continuum hypothesis is assumed then in both (b) and (c), the first assertion implies the second.

ProOF. $\quad(\mathrm{a}) \Longrightarrow(\mathrm{b})$ : Clearly, $B(X)$ is Dedekind complete, since $\bar{C}(X)$ is (A. 1). Second, since $M(X)$ is the order continuous dual of $\bar{C}(X)$ (A. 1 ) and since $l^{1}(X)$ is the order continuous dual of $B(X)$ (3.5), (a) implies that $M(X)=l^{1}(X)$. But this equality is equivalent to $X$ being scattered $[11,19.7 .6]$.

(b) $\Longrightarrow(\mathrm{c})$ : It follows from 3.5 that $\mathfrak{B}(X)=2^{X}$ (hence $B(X)=l^{\infty}(X)$ ), and from $[11,19.7 .6]$ that $M(X)=l^{1}(X)$.

$(\mathrm{c}) \Longrightarrow(\mathrm{d})$ : Considering the canonical imbedding $\tilde{\phi}: B(X) \rightarrow \bar{C}(X)$, the adjoint mapping $\tilde{\phi}^{\prime}$ transforms, by virtue of the assumption $M(X)=l^{1}(X)$, the order continuous dual $M(X)$ of $\bar{C}(X)$ onto the order continuous dual $l^{1}(X)$ of $B(X)$; by [7, Prop. 1] this means that $\tilde{\phi}$ is order continuous (in other words, if we think of $B(X)$ as a Riesz subspace of $\bar{C}(X)$ then a directed $(\leqq)$ majorized subset $A \neq \emptyset$ of $B(X)$ has the same least upper bound in $B(X)$ as it does in $\bar{C}(X)$.

This implies the topology $\mathfrak{I}$ on $B(X)$ to be a Fatou topology in the sense of [5]; clearly, since each $\mathfrak{T}$-bounded set of $B(X)$ is norm bounded (hence majorized), $\mathfrak{I}$ is also a Levi topology [5]. But $\mathfrak{B}(X)=2^{X}$ implies that $B(X)$ is Dedekind complete ; hence from Nakano's theorem [5, $23 \mathrm{~K}]$ it follows that $(B(X), \mathfrak{I})$ is complete (as a uniform space).

$(\mathrm{d}) \Longrightarrow(\mathrm{a})$ is trivial, since $B(X)$ is dense in $(\bar{C}(X), \mathfrak{I})$. Concerning the final assertion, we observe that by a long known result of BanachKuratowski (cf. [12, p. 150]) the continuum hypothesis implies that the cardinality of the continuum $I:=[0,1]$ is non-measurable (i. e., there exists no countably additive set function $\mu \neq 0$ on $2^{I}$ that vanishes on finite sets). Suppose, then, that $\mathfrak{B}(X)=2^{X}$ but $X$ is not scattered. There exists a continuous surjection $\tau: X \rightarrow I[11,8.5 .4]$. Now $\tau$ induces an isometric injection $f \rightarrow f \circ \tau$ of $C(I)$ into $C(X)$, with adjoint surjection 
$M(X) \rightarrow M(I)$. In particular, if $m$ denotes the Lebesgue-Borel measure [2] on $I$, there exists $\mu \in M(X)$ such that $m=\mu^{\circ} \tau^{-1}$. But since $\mathfrak{B}(X)=2^{X}$ it follows that $m$ has a countably additive extension to the power set $2^{I}$, vanishing on finite sets, which contradicts the non-measurability of $I$.

3. 7 NOTE. A shorter (but perhaps less instructive) proof of the equivalence of (a) through (d) in 3.6 results by observing that $(\mathrm{c}) \Longrightarrow(\mathrm{a})$, because (c) implies $\bar{C}(X)=M(X)^{\prime}=l^{1}(X)^{\prime}=l^{\infty}(X)$ and $B(X)=l^{\infty}(X)$; (a) $\Longrightarrow(\mathrm{d})$ follows from the completeness of $(\bar{C}(X), \mathfrak{I})$ which, in turn, rests on Grothendieck's theorem [9, IV. 6.2].

3. 8 REMARK. It is not known to the author if $X$ scattered implies $\mathfrak{B}(X)=2^{X}$. However, if $X$ is scattered with $X^{(\alpha)}$ void for some finite ordinal $\alpha$ then $\mathfrak{B}(X)=2^{X}$. The simplest example of this kind is the one point compactification of any discrete space; another is given by any compact (i. e., isolated) ordinal $\beta$ such that $\beta<\omega^{\omega}[11,8.6 .6]$.

\section{Literature}

[1] Aliprantis, C. D. and Burkinshaw, O., Locally Solid Riesz Spaces, New York: Academic Press 1978.

[2] BAUER, H., Wahrscheinlichkeitstheorie und Grundziige der Maßtheorie, 3. Aufl. Berlin-New York: W. de Gruyter 1978.

[ 3 ] Diestel, J. and UhL, J. J. Jr., Vector Measures. Providence, R. I.: Amer. Math. Soc. 1977.

[ 4 ] Dunford, N. and Schwartz, J. T., Linear Operators. Part I : General Theory. New York: Wiley 1958.

[5] Fremlin, D. H., Topological Riesz Spaces and Measure Theory. Cambridge University Press 1974.

[ 6 ] Halmos, P. R., Measure Theory. New York-Toronto-London: D. van Nostrand 1950.

[ 7 ] Schaefer, H. H., Dual Characterization of Order Continuity and Some Applications. Archiv d. Math. 51 (1988), 446-450.

[ 8 ] Schaefer, H. H., Radon, Baire, and Borel Measures on Compact Spaces I, Hokkaido Math. Journal Vol. 18 No. 2 (1989), 231-243.

[ 9 ] Schaefer, H. H., Topological Vector Spaces, $5^{\text {th }}$ print. New York-Berlin-HeidelbergTokyo : Springer 1986.

[10] Schaefer, H. H., Banach Lattices and Positive Operators. Berlin-Heidelberg-New York : Springer 1974.

[11] Semadeni, Z., Banach Spaces of Continuous Functions I, Polish Scientific Publishers: Warszawa 1971.

[12] ZaAnen, A. C., Riesz Spaces II. Amsterdam-New York-Oxford: North Holland 1983.

Mathematisches Institut der Universität Tübingen Auf der Morgenstelle 10 7400 Tubingen, F. R. G. 